\title{
Strategies Towards Improving Clinical Outcomes of Peptide Receptor Radionuclide Therapy
}

\author{
N.S. Minczeles ${ }^{1,2,3} \cdot$ J. Hofland ${ }^{1,3} \cdot$ W.W. de Herder ${ }^{1,3} \cdot$ T. Brabander ${ }^{2,3}$ \\ Accepted: 26 January 2021 / Published online: 15 March 2021 \\ (C) The Author(s) 2021
}

\begin{abstract}
Purpose of Review Peptide receptor radionuclide therapy (PRRT) with $\left[{ }^{177} \mathrm{Lu}\right.$-DOTA $\left.{ }^{0}, \mathrm{Tyr}^{3}\right]$ octreotate is an effective and safe second- or third-line treatment option for patients with low-grade advanced gastroenteropancreatic (GEP) neuroendocrine neoplasms (NEN). In this review, we will focus on possible extensions of the current use of PRRT and on new approaches which could further improve its treatment efficacy and safety.

Recent Findings Promising results were published regarding PRRT in other NENs, including lung NENs or high-grade NENs, and applying PRRT as neoadjuvant or salvage therapy. Furthermore, a diversity of strategic approaches, including dosimetry, somatostatin receptor antagonists, somatostatin receptor upregulation, radiosensitization, different radionuclides, albumin binding, alternative renal protection, and liver-directed therapy in combination with PRRT, have the potential to improve the outcome of PRRT. Also, novel biomarkers are presented that could predict response to PRRT.

Summary Multiple preclinical and early clinical studies have shown encouraging potential to advance the clinical outcome of PRRT in NEN patients. However, at this moment, most of these strategies have not yet reached the clinical setting of randomized phase III trials.
\end{abstract}

Keywords Peptide receptor radionuclide therapy $\cdot$ Neuroendocrine tumour

\section{Introduction}

Peptide receptor radionuclide therapy (PRRT) with $\left[{ }^{177} \mathrm{Lu}-\right.$ DOTA $^{0}$, Tyr $\left.^{3}\right]$ octreotate $\left({ }^{177}\right.$ Lu-DOTATATE or ${ }^{177} \mathrm{Lu}-$ oxodotreotide) is registered for the treatment of progressive and advanced grade 1-2 gastroenteropancreatic (GEP) and thymic and bronchial (in the USA) neuroendocrine tumours (NETs). Patients are treated with intravenously administered somatostatin analogues (SSAs) that are coupled to radionuclides, such as

This article is part of the Topical Collection on Neuroendocrine Neoplasms

T. Brabander

t.brabander@erasmusmc.nl

1 Department of Internal Medicine, Section of Endocrinology, Erasmus MC and Erasmus MC Cancer Center,

Rotterdam, The Netherlands

2 Department of Radiology \& Nuclear Medicine, Erasmus MC, Rotterdam, The Netherlands

3 ENETS Center of Excellence Rotterdam, Rotterdam, The Netherlands
Lutetium-177 or Yttrium-90. These radiolabelled SSAs can target the somatostatin receptor (SSTR) subtypes on the tumour cell surface. Via these SSTRs, the radiolabelled SSAs are internalized into the cell and cause DNA damage in the cell nucleus which subsequently leads to cell death.

Neuroendocrine neoplasms (NENs) are a heterogeneous disease that can be categorized according to the anatomical site of the primary tumour (e.g. GEP, bronchial, thymic, or foregut, midgut, hindgut), the disease stage, the tumour grade and differentiation, and according to hormonal hypersecretion. In NEN, which are known to highly express SSTRs, PRRT is considered an effective and safe treatment option. The phase III NETTER-1 randomized controlled trial demonstrated that in low- to intermediate grade metastatic midgut NET patients with progressive disease using first-line long-acting octreotide, ${ }^{177} \mathrm{Lu}$ DOTATATE therapy combined with long-acting octreotide significantly prolonged progression-free survival (PFS), likely also overall survival (OS) [1••], and improved quality of life [2] compared to doubling the dose of octreotide therapy. In a large meta-analysis, the objective response (OR) rate (complete response (CR) or partial response (PR)) was 35\% and the disease control rate (CR or PR or stable disease (SD)) was $83 \%$ in NEN 
patients treated with PRRT [3]. Following the results of PRRT in NEN patients, other applications of theranostics are under investigation for different types of malignancies, such as ${ }^{177}$ Lu-PSMA-617 for castration-resistant prostate carcinoma, ${ }^{177} \mathrm{Lu}-\mathrm{NeoBOMB} 1$ for gastrin-releasing peptide receptorpositive tumours, and different radiolabelled antibodies for haematological and lymphoid malignancies [4].

In this review, we will elaborate on possible extensions of the current use of PRRT with ${ }^{177} \mathrm{Lu}$-DOTATATE, e.g. the place of PRRT in the treatment sequence of NEN, treatment with PRRT in other types of NEN, or in high-grade NEN. Furthermore, since a proportion of patients treated with ${ }^{177}$ Lu-DOTATATE does not show disease control or disease response [3], new strategies that could further improve the treatment efficacy are highlighted. Lastly, new research lines that try to decrease the risk of treatment-related toxicity are discussed. An overview of these topics is provided in Fig. 1.

\section{Other Indications}

\section{Non-GEP NEN}

Treatment with ${ }^{177} \mathrm{Lu}$-DOTATATE is in most countries registered for advanced SSTR-positive low-grade GEP NENs (Ki67 index $\leq 20 \%$ ), but it may also be an effective therapy in other SSTR-expressing tumours, such as paraganglioma (PGL) and phaeochromocytoma (PCC), thyroid carcinoma, and meningioma. Furthermore, ${ }^{177}$ Lu-DOTATATE therapy is also registered for thymic and bronchial NENs in the USA. In approximately $25 \%$ of the NEN patients, the primary tumour arises from the bronchopulmonary system [5]. These lung NENs are categorized as typical and atypical carcinoids [6]. Various studies have determined the efficacy of PRRT in bronchial NENs. Following treatment with ${ }^{177} \mathrm{Lu}-$ DOTATATE, the OR rate ranged $10-33 \%$ and the SD rate ranged $30-63 \%$ in patients with bronchial NENs [7-12]. The median PFS of PRRT ranged from 19 to 31 months across different series [7, 8, 10, 11].

Patients with PGL, which arise from the chromaffin cells of the autonomous nervous system, and PCC, which arise from the adrenal medulla, have been treated with PRRT as well. In our study, ${ }^{177} \mathrm{Lu}$-DOTATATE therapy in 27 PGL and 3 PCC patients, with $67 \%$ of them having progressive disease at baseline, resulted in PR and SD in $23 \%$ and $67 \%$ of the patients, respectively. Median PFS of the total study population was 30 months but was increased to 91 months in the subgroup of patients with parasympathetic PGL [13]. In another study involving ${ }^{177}$ Lu-DOTATATE therapy in 13 PGL and 9 PCC patients, the PR and SD rates were $9 \%$ and $91 \%$, respectively. Of those patients, $41 \%$ had, prior to PRRT, progressive disease after previous therapies, and 59\% received first-line PRRT, mainly because of symptomatic disease. In this study, the median PFS was 22 months [14].

Although the data comprising these non-GEP NEN are mostly obtained from heterogeneous retrospective studies with limited numbers of patients, they suggest that PRRT could have a place in the treatment algorithm for bronchopulmonary NEN, PGL, and PCC.

\section{High-Grade NEN}

According to the proliferation index (Ki67\%), mitotic count, and histology, GEP NENs are classified as well differentiated NETs (grade 1; Ki67\% < 3\%, grade 2; Ki67\% 3-20\%, and grade $3 ; \mathrm{Ki} 67 \%>20 \%$ ), or poorly differentiated small-cell or large-cell neuroendocrine carcinoma (NEC; Ki67\% > 20\%) [15]. Tumour grading is one of the most relevant, independent prognostic factors in NEN patients [16]. PRRT can play a role in the treatment of high-grade NENs, even though these tumours are more aggressive. However, the expression of SSTRs on the cell surface can vary, and sufficient expression is needed for treatment with PRRT. Grade 3 NETs and NECs express SSTRs in 87-92\% and 37-50\%, respectively [17-20].

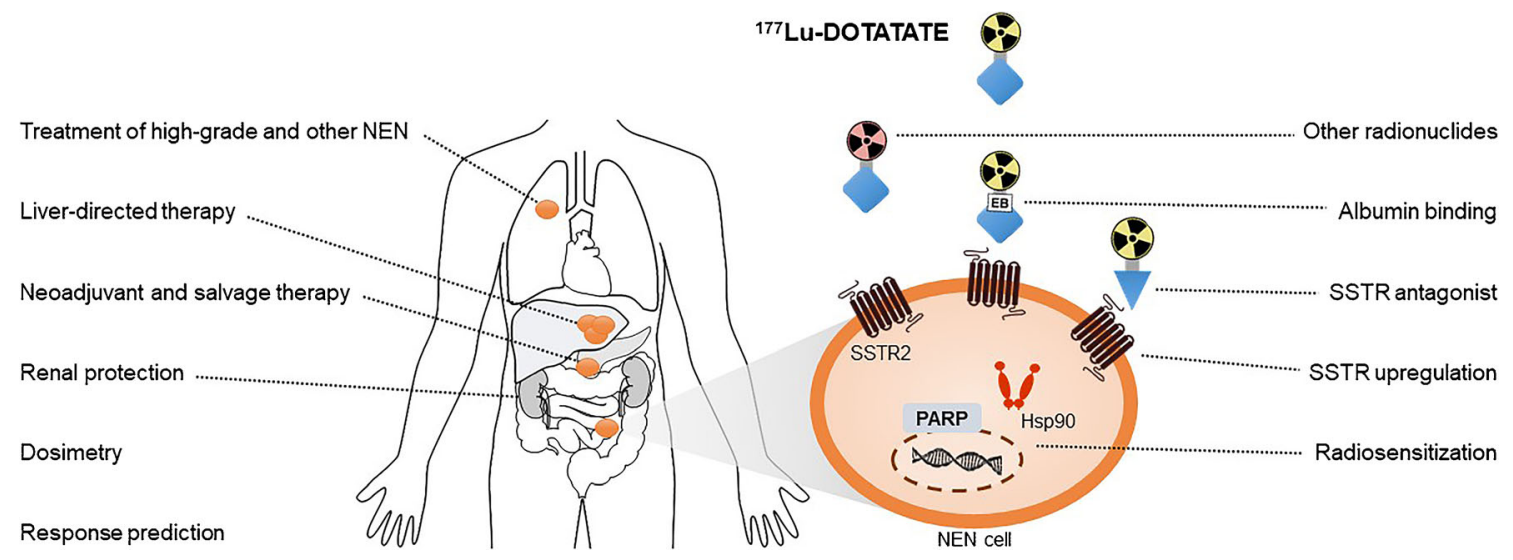

Fig. 1 Overview of future possibilities that could extent the use of PRRT or could improve the efficacy and safety of PRRT. NEN neuroendocrine neoplasm, SSTR somatostatin receptor, $H s p 90$ heat shock protein $90, P A R P$ poly(ADP-ribose) polymerase 
Several studies report on the use of PRRT in these patient categories [21-24]. The outcomes of the largest retrospective, multicentre, analysis of 149 grade 3 GEP NET and NEC patients treated with ${ }^{177} \mathrm{Lu}$-DOTATATE, ${ }^{90} \mathrm{Y}$-DOTATOC or a combination of these two radiotherapeuticals were comparable to these smaller studies. In 114 evaluable patients, an OR rate of $42 \%$ was reported, and disease control was reported in $69 \%$ of the patients with documented disease progression before start of PRRT. The median PFS was 19 months in grade 3 NET, 11 months in NEC with a Ki67\% of $21-54 \%$, and 4 months in NEC with a $\mathrm{Ki} 67 \%$ of $\geq 55 \%$. The median OS in grade 3 NET, NEC (Ki67\% of 21-54\%), and NEC (Ki67\% of $\geq 55 \%$ ) was 44 months, 22 months, and 9 months, respectively. Treatment toxicity was limited [25••]. Therefore, PRRT could be a valuable treatment option in grade 3 NET and NEC. The NETTER-2 randomized controlled trial is currently enrolling patients to investigate the efficacy of first-line PRRT with ${ }^{177}$ Lu-DOTATATE in patients with advanced grade 2 or 3 GEP NET.

\section{Treatment Sequence}

Although the benefit of PRRT as a second- or third-line palliative therapeutic option for grade 1-2 GEP NET has been established, the best timing in the treatment sequence is still debated. In patients with advanced grade 1-2 GEP NET, PRRT is positioned among other management options of locoregional therapy and anti-proliferative drugs, such as everolimus, and for pancreatic NEN (panNEN) cytotoxic chemotherapy and sunitinib [26]. A phase III randomized controlled trial (COMPETE, NCT03049189) will compare the efficacy and safety of PRRT with $\left[{ }^{177} \mathrm{Lu}-\mathrm{DOTA}{ }^{0}, \mathrm{Tyr}^{3}\right]$ octreotide ( ${ }^{177}$ Lu-DOTATOC) with everolimus in grade $1-2$ GEP NET patients. We will further discuss other options for PRRT in the treatment algorithm, such as first-line and neoadjuvant, and retreatment with PRRT.

\section{First-Line and Neoadjuvant}

Given its potent effects with response rates up to $39 \%$ in all NEN and up to $54 \%$ in panNEN [7•], first-line PRRT constitutes a potential option for treatment-naïve patients with bulky disease or severe hormonal symptoms. In a recent publication including 45 patients with an advanced NEN who received first-line ${ }^{177} \mathrm{Lu}$-DOTATATE, a 30\% PR rate was found. In this study, PRRT was combined with oral capecitabine, suggesting that the addition of this chemotherapeutic agent conferred no extra benefit in response rates [27]. It is also important to realize that first-line PRRT in an experimental prospective setting already started in many European and Australian centres years before the results of the PROMID and CLARINET studies were published. These studies led to the registration of first-line SSA therapy in grade 1-2 midgut NETs after 2009 [28] and in GEP NETs in 2014 [29].

Early PRRT can also be applied for downstaging or as neoadjuvant treatment in order to make surgical tumour resection possible or improve its curation rates. Neoadjuvant PRRT has been described most frequently in panNEN [30-33]. We demonstrated that out of the 29 borderline resectable or unresectable panNEN patients, 9 patients underwent successful surgery after ${ }^{177}$ Lu-DOTATATE [32]. The most recent retrospective analysis consisted of 23 resectable or potentially resectable panNEN patients who were treated with Yttrium-90 and/or Lutetium-177 labelled somatostatin analogues followed by surgical resection. These patients were matched to 23 panNEN patients who underwent upfront curative surgery. Neoadjuvant PRRT could lower the risk of postoperative pancreatic fistula and the number of tumour-positive lymph nodes. In addition, as a result of PRRT, less patients had tumour involvement of the superior mesenteric vein or portal vein [33]. Tumour compression or invasion of important venous and arterial structures can preclude upfront curative resection, so there is an unmet need for a therapeutic strategy to render these panNENs resectable. Neoadjuvant PRRT seems to be promising in this respect. However, larger, prospective, trials are needed to establish a PFS and OS benefit for patients that undergo surgery after neoadjuvant PRRT.

\section{Salvage}

When NEN patients have progressive disease after PRRT, few systemic treatment options are available [26]. Small retrospective studies have illustrated that retreatment with PRRT may be a safe and efficient strategy [34-40]. However, different treatment regimens regarding dose, number of cycles, and choice of radionuclide were used. Also, not every study included the same criteria regarding initial treatment response (e.g. PFS after the first PRRT). Recently, we published the largest GEP and bronchial NEN patient cohort receiving salvage PRRT with ${ }^{177} \mathrm{Lu}$-DOTATATE. Inclusion criteria for salvage PRRT consisted of a PFS of $\geq 18.0$ months from start of initial PRRT. After salvage PRRT with two cycles of ${ }^{177}$ Lu-DOTATATE, $16 \%$ and $60 \%$ of the patients had an OR or SD, respectively, with a median PFS of 15 months. Following re-retreatment with PRRT (i.e. two cycles of

${ }^{177}$ Lu-DOTATATE after a PFS of $\geq 14.0$ months after the first retreatment with PRRT), observed OR and SD rates were $39 \%$ and $54 \%$, respectively, and the median PFS was 14 months. Compared to a non-randomized control group, salvage PRRT resulted in a significantly longer OS. We did not find a higher incidence of renal insufficiency, myelodysplastic syndrome or acute myeloid leukaemia compared to initial treatment with PRRT $[41 \bullet \bullet]$. 


\section{Treatment Optimization}

There are several components of PRRT that could be altered in order to optimize the radiation-induced cytotoxic effect on tumour cells and improve its safety.

\section{Dosimetry}

In panNEN patients, a correlation was illustrated between the absorbed tumour dose and the tumour response following ${ }^{177}$ Lu-DOTATATE [42], but this was not found in small intestinal NEN [43]. On the contrary, the cumulative administered activity does seem to correlate with tumour response in small intestinal NEN [43] and with PFS in panNEN patients [44]. As the absorbed tumour dose varies interindividually, dosimetry-guided treatments are under investigation with the aim to increase the efficacy and safety of PRRT by varying the administered activity. Two prospective trials that consisted of NEN patients treated with a personalized dose have been reported. In the first study, in which dosimetry was based on the bone marrow dose (maximum allowed absorbed dose of $2 \mathrm{~Gy}$ ) and kidney dose (maximum allowed absorbed dose of $23 \mathrm{~Gy}$ ), approximately half of the patients received more than the standard four cycles of $7.4 \mathrm{GBq}{ }^{177} \mathrm{Lu}$-DOTATATE. In the patients reaching the 23 Gy kidney dose, a median PFS of 33 months and a median OS of 54 months were observed compared to a median PFS of 15 months and a median OS of 25 months in patients in whom the maximum kidney dose was not reached [45•]. In another study, in which the administered activity of ${ }^{177} \mathrm{Lu}$-DOTATATE per cycle was varied based on the glomerular filtration rate, body surface area, and renal dosimetry, it was shown that personalized doses augmented the absorbed tumour dose with a median 1.26-fold and resulted in a PR or minor response rate of 59\% and a SD rate of $33 \%$ [46]. In both studies, the risk of severe renal or bone marrow toxicity was not increased [45, 46]. At this moment, dose adjustments based on individual dosimetry are not implemented in clinical practice for the standard four cycles of PRRT, because the standard treatment is considered as safe $[1,7]$. However, IAEA, EANM, and SNMMI guidelines do recommend a form of dosimetry in every patient, usually a posttherapy scan after $24 \mathrm{~h} \mathrm{[47].}$

\section{Liver-Directed Treatment}

GEP NENs predominantly metastasize to the liver [48]. Local liver-directed treatment, including resection, bland or chemoembolization (TACE), radioembolization (SIRT), radiofrequency ablation (RFA), microwave and cryoablation, highintensity focused ultrasound (HIFU), laser, brachytherapy, and irreversible electroporation (IRE) can be options depending on the availability at the institution. In case of diffuse metastatic liver lesions, systemic treatment, liver-directed treatment or the combination can be considered [26]. In the HEPAR PluS trial, systemic PRRT was followed by additional SIRT using Holmium-166 microspheres in 31 grade 1 or 2 NET patients with at least three unresectable liver metastases. At 3-month follow-up, liver-specific and whole-patient OR rates were achieved in $43 \%$ and $40 \%$ of the patients, respectively. Short-term toxicity did not seem to be worse as compared to PRRT alone [49॰].

Another approach with regard to enhancing the radiation dose delivered to liver metastases is intra-arterial PRRT administered via the hepatic artery. Limited data indicated an 1.06- to 9.2-fold increased uptake in liver metastases after intra-arterial administration of somatostatin analogues labelled with Indium-111, Gallium-68 or Lutetium-177 compared to intravenous administration [50]. In a recent pilotstudy, however, no increased tumour uptake after intraarterial ${ }^{68}$ Ga-DOTATOC injection was observed [51]. The LUTIA trial is poised to investigate the absorbed tumour dose and response of intra-arterial ${ }^{177} \mathrm{Lu}$-DOTATATE in progressive, liver-dominant, and unresectable NEN patients [52].

\section{Somatostatin Analogues}

The anti-proliferative effect of SSAs was established in the PROMID [28] and CLARINET [29] trials. However, it is not yet well established whether SSAs should be combined with or added to PRRT for increasing the response and improving survival. In clinical practice, PRRT monotherapy, PRRT in combination with a long-acting SSA with or without a short-acting SSA, and PRRT followed by SSA maintenance therapy are all applied. In the NETTER-1 trial, ${ }^{177} \mathrm{Lu}$ DOTATATE in combination with $30 \mathrm{mg}$ octreotide LAR per 4 weeks was superior to $60 \mathrm{mg}$ octreotide LAR per 4 weeks, but no control group of PRRT alone was included $[1 \bullet \cdot]$. Long-acting SSAs appeared to decrease the uptake in the liver and spleen on SSTR imaging. However, an increase in tumour uptake was not observed in all studies [53-56]. Thus, it is unclear whether pretreatment with a long-acting SSA results in a higher tumour dose after PRRT. One retrospective study compared PRRT alone with PRRT in combination with SSA (combined and/or as maintenance after PRRT). The authors reported higher tumour response rates (OR $63 \%$ vs. $40 \%$ ) and a longer PFS (48 months vs. 27 months) and OS (91 months vs. 47 months) for the PRRT with SSA group, especially in patients with a higher Ki67\%, higher tumour burden, and with hormone-secreting tumours [57].

\section{Somatostatin Receptor Antagonists}

Radionuclide-coupled SSAs are internalized into tumour cells after coupling to the SSTRs expressed on the tumour cell membrane. It was postulated that this mechanism would 
facilitate an optimal dose of radioactivity to the cell nucleus. SSTR antagonists, on the other hand, rarely internalize into SSTR-positive tumour cells [58, 59], but, because they have a higher affinity, they bind to more SSTRs than the agonists [59, 60]. Preclinical in vitro and in vivo studies with different radiolabelled SSTR antagonists demonstrated that, as a result, PRRT with SSTR antagonists led to a higher tumour uptake than with SSTR agonists [58, 59, 61]. The higher tumour dose achieved after administration of the antagonist ${ }^{177} \mathrm{Lu}$-DOTAJR11 $\left({ }^{177} \mathrm{Lu}\right.$-OPS201) compared to the agonist ${ }^{177} \mathrm{Lu}-$ DOTATATE was demonstrated in a pilot study in four NEN patients [62]. Furthermore, in a phase I study involving 20 NEN patients treated with one or two cycles of $7.4 \mathrm{GBq}$ ${ }^{177}$ Lu-DOTA-JR 11 ( ${ }^{177} \mathrm{Lu}-\mathrm{OPS} 201$ ), encouraging response rates $(5 \% \mathrm{CR}, 40 \% \mathrm{PR}$, and $40 \% \mathrm{SD}$ ) were reported. However, in 4 of 7 patients, grade 4 hematologic toxicity occurred after the second treatment cycle, so for the remaining patients, a dose reduction for a maximum bone marrow dose of 1 Gy was needed [63]. Ongoing trials (NCT02592707, NCT02609737) are further investigating the efficacy and toxicity of ${ }^{177}$ Lu-DOTA-JR11 ( ${ }^{177}$ Lu-OPS201) in NEN patients.

\section{Somatostatin Receptor Upregulation}

As PRRT depends on the binding of SSTRs, increasing the number of these receptors on the tumour cell membrane is a potential target for improving the efficacy of PRRT. Furthermore, when SSTR expression can be successfully stimulated, PRRT could also be a treatment option for patients with limited uptake on SSTR imaging. Several preclinical studies reported promising results regarding upregulation of the SSTR subtype 2 (SSTR2) expression by using epigenetic modifiers. SSTR2 expression was enhanced by administration of DNA methyltransferase inhibitors [64-67] and histone deacetylase inhibitors [64-71]. Following treatment with epigenetic drugs in NEN cells, increased apoptosis-induced cell death $[67,68,71]$, decreased proliferative activity [68], and augmented radiosensitivity [67] have also been reported. Synergistic effects on SSTR2 upregulation were observed for the combination of a DNA methyltransferase inhibitor with a histone deacetylase inhibitor [64, 65, 67]. These stimulatory effects of epigenetic drugs on SSTR2 expression have been confirmed in NEN xenograft-bearing mice $[65,69,70]$, but these drugs have not been tested in clinical trials in NEN patients yet.

\section{Albumin Binding}

Another strategy for enhancing the tumour uptake of Lutetium-177 is increasing its bioavailability by extending the biological half-life of the radiolabelled somatostatin analogue in blood. This has been executed by combining ${ }^{177} \mathrm{Lu}-$ DOTATATE with an Evans blue structure, a reversible albumin binder $[72,73]$. In the first small clinical study, in which metastatic NEN patients were given a single low dose of ${ }^{177}$ Lu-DOTA-EB-TATE $(n=5)$ or ${ }^{177}$ Lu-DOTATATE $(n$ $=3$ ), a 7.9-fold increase in tumour dose was observed in the patients receiving ${ }^{177} \mathrm{Lu}$-DOTA-EB-TATE [74]. Hereafter, singe doses of $1.11 \mathrm{GBq}, 1.85 \mathrm{GBq}$, and $3.7 \mathrm{GBq}{ }^{177} \mathrm{Lu}-$ DOTA-EB-TATE were compared with $3.7 \mathrm{GBq}{ }^{177} \mathrm{Lu}-$ DOTATATE in 33 metastatic NEN patients with high uptake on ${ }^{68} \mathrm{Ga}$-DOTATATE PET/CT. Regarding the decrease in post-treatment maximum standardized uptake value (SUV) and response rates, the higher doses of ${ }^{177}$ Lu-DOTA-EBTATE were more effective than ${ }^{177}$ Lu-DOTATATE [75]. The decrease in the maximum SUV was also observed after treatment with up to 3 cycles with a cumulative dose of 5.7 $\mathrm{GBq}$ and $10.5 \mathrm{GBq}{ }^{177}$ Lu-DOTA-EB-TATE [76••]. A decrease in SUV, however, is not validated as a treatment response indicator [77-79]. Reported disease control rates, assessed by the EORTC criteria, were $67 \%, 83 \%$, and $72 \%$ for the cumulative doses of $3.5 \mathrm{GBq}, 5.7 \mathrm{GBq}$, and $10.5 \mathrm{GBq}$ ${ }^{177}$ Lu-DOTA-EB-TATE, respectively. Short-term grade 3 hepatotoxicity occurred in 2 patients $(6 \%)$, and grade 3 haematological toxicity occurred in 4 patients (13\%), of whom 3 patients were pretreated with chemotherapy or targeted therapy $\left[76 \bullet^{\circ}\right]$. Nevertheless, the prolongation of the half-life and consequently increase of the radiation dose in kidneys and bone-marrow raises concerns about toxicity.

\section{Radiosensitization}

Several options have been investigated in order to increase the efficacy of PRRT by rendering the cell more sensitive to radiation. One strategy concerns the inhibition of the DNA repair mechanism that repairs the DNA damage induced by the beta-emitting radiation of PRRT. The DNA damage consists of single- and double-strand DNA breaks, and the singlestrand DNA breaks can be repaired by Poly(ADP-ribose) polymerase-1 (PARP). In vitro administration of a PARP inhibitor together with ${ }^{177} \mathrm{Lu}$-DOTATATE induced more double-strand breaks $[80,81]$ and increased cell death $[80$, 82] compared to PRRT alone. In vivo experiments in murine xenograft models furthermore showed prolonged inhibition of tumour growth (34 days vs. 14 days) and a longer median survival (44 days vs. 37 days) for the combination of PRRT and a PARP inhibitor versus PRRT alone [81].

Another method of increasing the radiosensitivity of the tumour cells is by combining PRRT with a heat shock protein 90 (Hsp90) inhibitor. As a molecular chaperone, Hsp90 is involved in folding, remodelling, and stabilization of, among other proteins, proteins that are associated with oncogenesis [83]. Moreover, Hsp90 is upregulated in small intestinal NEN cells. In vitro (in NEN cell lines), ex vivo (in patient-derived tumour tissues), and in vivo (in mice with xenografted tumours), the addition of the Hsp90 inhibitor ganetespib to 
${ }^{177} \mathrm{Lu}$-DOTATATE therapy resulted in a better anti-tumour response [84]. Another Hsp90 inhibitor, onalespib, enhanced the in vivo treatment effect of PRRT as well. It also induced the expression of Hsp 70, which could reduce the renal toxicity [85]. No clinical trials have been performed in NEN patients or in combination with PRRT yet.

\section{Other Radiotherapeuticals}

Currently, PRRT is conducted with the beta-emitters Lutetium-177 or Yttrium-90. Radionuclides that emit higher levels of energy, such as the alpha-emitting Actinium-225, Bismuth-213, and Lead-212, have the potential to cause more radiation damage to the tumour cells and thereby to increase the efficacy of PRRT. In a prospective study, including 32 metastatic GEP NEN patients previously treated with ${ }^{177} \mathrm{Lu}-$ DOTATATE, patients were treated with a maximum cumulative dose of $55.5 \mathrm{MBq}^{225} \mathrm{Ac}$-DOTATATE. Twenty-four patients were assessed in the interim analysis. In the patients with SD after ${ }^{177} \mathrm{Lu}$-DOTATATE, $67 \%$ had PR after ${ }^{225}$ AcDOTATATE. In the patients with progressive disease after ${ }^{177}$ Lu-DOTATATE, PR and SD after ${ }^{225}$ Ac-DOTATATE were achieved in $58 \%$ and $42 \%$, respectively. No grade 3 or 4 renal, liver or bone-marrow toxicity occurred [86 $6^{\circ}$. Longterm follow-up is needed to reveal whether these response rates also lead to a longer PFS and OS, without increased toxicity. A retrospective analysis with a longer follow-up also described encouraging results after treatment with intraarterial administration of ${ }^{213} \mathrm{Bi}$-DOTATOC in 7 NEN patients, pretreated with ${ }^{90} \mathrm{Y}$ - or ${ }^{177} \mathrm{Lu}$-DOTATOC. However, an overall decline of the renal function was detected, chronic anaemia developed in 3 patients, and acute myeloid leukaemia was diagnosed in one patient 2 years after ${ }^{213} \mathrm{Bi}$-DOTATOC [87]. Preclinical research has also focused on ${ }^{212} \mathrm{~Pb}$ DOTAMTATE, which was capable of decreasing tumour volumes and prolonging survival in mice [88]. ${ }^{212} \mathrm{~Pb}$ DOTAMTATE is currently being investigated in a phase I trial (NCT03466216). Another beta-emitting radionuclide, Copper-67, is of interest as well. ${ }^{67} \mathrm{Cu}$-SARTATE was equally effective in vivo as ${ }^{177}$ Lu-DOTATATE [89], but Copper has the advantage that it can be implemented for both diagnostic (with Copper-64) and therapeutic (with Copper-67) purposes.

\section{Nephrotoxicity}

Severe impaired renal function is an exclusion criterion for PRRT. Furthermore, renal protection during PRRT is provided by the concurrent infusion of an amino acid combination, preventing the reabsorption of the radiopeptides in the proximal tubular cells. No severe subacute renal toxicity has been observed after ${ }^{177} \mathrm{Lu}$-DOTATATE administration with concomitant amino acid infusion and exclusion of patients with a creatinine level of $\leq 150 \mu \mathrm{mol} / \mathrm{L}$ or a clearance of $<40 \mathrm{~mL} / \mathrm{min}$.
Long-term grade 3 toxicity occurred in $1 \%$ of the treated patients, and no long-term grade $\geq 4$ toxicity was reported. It was estimated that the glomerular filtration rate declines annually with $3 \%$, with $2.4 \%$ of the patients having an annual loss of more than $10 \%$ [90]. Nephrotoxicity occurs more frequently after PRRT with Yttrium-90 than PRRT with Lutetium-177 [91]. A new renal protective agent, the human protein $\alpha_{1}$-microglobulin, is currently under investigation. Radiation can affect tumour-surrounding cells by induced oxidative stress. $\mathrm{A}_{1^{-}}$ microglobulin is an antioxidation and radical scavenger protein [92] that is, after intravenous administration, mostly localized in the kidneys [93]. Preclinical experiments demonstrated that the administration of the $\alpha_{1}$-microglobulin in ${ }^{177} \mathrm{Lu}$ DOTATATE-treated mice reduced the radiation damage of the kidneys [94], without affecting the treatment efficacy [95].

\section{Treatment Response Prediction}

Since the disease control rate of PRRT in NEN patients is $83 \%$ [3], a minority of patients do not respond to treatment with PRRT and are at risk for its adverse events and ongoing disease progression. Therefore, reliable biomarkers are needed to predict an individual patient's response to PRRT.

\section{PRRT Predictive Quotient}

The PRRT predictive quotient (PPQ) was developed by linking pretreatment gene clusters in circulating RNA to clinical and tumour characteristics of patients who responded to PRRT compared to patients who did not respond to PRRT. After including significant factors, consisting of blood levels of expression of genes involved in growth factor signalling and metabolism, and histological grade, the PPQ was found to accurately predict response to PRRT in 94\% of the patients [96]. In two prospective validation cohorts, non-responders were correctly predicted in 93\% and $100 \%$ and responders in $94 \%$ and $97 \% 6$ to 9 months after completing PRRT [97]. However, non-responders still displayed a median PFS which was similar to other registered therapies for advanced NEN, questioning whether PRRT should be withheld on the basis of this biomarker.

\section{Imaging}

One of the eligibility criteria for PRRT is sufficient tumour uptake on SSTR imaging, i.e. ${ }^{68} \mathrm{Ga}$-DOTATATE/-TOC PET/ CT or ${ }^{111}$ In-DTPA-octreotide scintigraphy, because the uptake reflects the presence of SSTRs. The uptake of the tumour related to the uptake of the liver, kidneys, and spleen on ${ }^{111}$ InDTPA-octreotide scintigraphy (also known as the Krenning scale) was found to correlate with OR following treatment with PRRT [98]. Efforts have been made to determine parameters on pretherapeutic ${ }^{68} \mathrm{Ga}$-DOTATATE/-TOC PET/CT that 
predict response to PRRT as well. A predictive parameter seems to be the SUV, although varying maximum SUV thresholds have been proposed [79, 99-101], and these findings were not confirmed in all studies [78, 102]. Another functional imaging feature of importance may be the SSTR heterogeneity of the tumour, as this seems to correlate with morphological response [103], time to progression [104, 105], and OS [104-106] following treatment with PRRT.

\section{Conclusions}

Peptide receptor radionuclide therapy is an approved medical treatment option for grade 1-2 GEP NETs that are progressive after first- or second-line treatment agents. Outcomes in terms of response, PFS and OS in this patient group are favourable. PRRT is not registered for all NEN patient subcategories, such as high-grade NEN (grade 3 NET and NEC) or-in most countries - bronchopulmonary NEN, although promising results in these patient categories have been reported as well. The same applies for treatment with PRRT in first-line, neoadjuvant or salvage setting. Unfortunately, in this field, data from randomized phase III trials are lacking. Furthermore, many approaches are currently under investigation aiming for optimizing the efficacy and safety of PRRT or finding parameters that could predict response to PRRT. Encouraging results were published regarding dosimetry, SSTR antagonists, radiosensitization, alphaemitting radionuclides, albumin binding, SSTR upregulation, and liver-directed therapy in combination with PRRT. Unfortunately, many of these studies are performed in small heterogeneous patient populations or are still in a preclinical stage. Further clinical trials are necessary to demonstrate superiority compared to standard therapy and to evaluate toxicity in these new treatments.

\section{Declarations}

Conflict of Interest Noémie S. Minczeles declares that she has no conflict of interest. Johannes Hofland has received compensation from Advanced Accelerator Applications / Novartis and Ipsen for service on advisory boards, and has received speaker's honorarium from Ipsen. Wouter W. de Herder has received research funding from Ipsen; has received speaker's honoraria from Advanced Accelerator Applications / Novartis, Ipsen, Pfizer, and Sanofi; and has received compensation from Advanced Accelerator Applications / Novartis and Ipsen for service on advisory boards. Tessa Brabander has received compensation from Advanced Accelerator Applications / Novartis for service on an advisory board, and has received speaker's honoraria from Advanced Accelerator Applications / Novartis and Medscape.

Human and Animal Rights and Informed Consent All reported studies/ experiments with human or animal subjects performed by the authors have been previously published and complied with all applicable ethical standards (including the Helsinki declaration and its amendments, institutional/national research committee standards, and international/national/institutional guidelines).

Open Access This article is licensed under a Creative Commons Attribution 4.0 International License, which permits use, sharing, adaptation, distribution and reproduction in any medium or format, as long as you give appropriate credit to the original author(s) and the source, provide a link to the Creative Commons licence, and indicate if changes were made. The images or other third party material in this article are included in the article's Creative Commons licence, unless indicated otherwise in a credit line to the material. If material is not included in the article's Creative Commons licence and your intended use is not permitted by statutory regulation or exceeds the permitted use, you will need to obtain permission directly from the copyright holder. To view a copy of this licence, visit http://creativecommons.org/licenses/by/4.0/.

\section{References}

Papers of particular interest, published recently, have been highlighted as:

- Of importance

- Of major importance

1.• Strosberg J, El-Haddad G, Wolin E, Hendifar A, Yao J, Chasen B, et al. Phase 3 trial of (177)Lu-dotatate for midgut neuroendocrine tumors. N Engl J Med. 2017;376(2):125-35. https://doi.org/10. 1056/NEJMoa1607427. The phase 3 randomized controlled trial demonstrated that in patients with low- to intermediate grade metastatic midgut NET, ${ }^{177}$ Lu-DOTATATE therapy combined with long-acting SSA significantly prolongs PFS, and likely also OS, compared to high dose octreotide therapy.

2. Strosberg J, Wolin E, Chasen B, Kulke M, Bushnell D, Caplin M, et al. Health-related quality of life in patients with progressive midgut neuroendocrine tumors treated with (177)Lu-dotatate in the phase III netter-1 trial. J Clin Oncol. 2018;36(25):2578-84. https://doi.org/10.1200/JCO.2018.78.5865.

3. Wang LF, Lin L, Wang MJ, Li Y. The therapeutic efficacy of 177Lu-DOTATATE/DOTATOC in advanced neuroendocrine tumors: A meta-analysis. Medicine (Baltimore). 2020;99(10): e19304. https://doi.org/10.1097/MD.0000000000019304.

4. Sgouros G, Bodei L, McDevitt MR, Nedrow JR. Radiopharmaceutical therapy in cancer: clinical advances and challenges. Nat Rev Drug Discov. 2020;19(9):589-608. https://doi.org/10.1038/s41573-0200073-9.

5. Yao JC, Hassan M, Phan A, Dagohoy C, Leary C, Mares JE, et al. One hundred years after "carcinoid": epidemiology of and prognostic factors for neuroendocrine tumors in 35,825 cases in the United States. J Clin Oncol. 2008;26(18):3063-72. https://doi.org/ 10.1200/JCO.2007.15.4377.

6. Rindi G, Klimstra DS, Abedi-Ardekani B, Asa SL, Bosman FT, Brambilla E, et al. A common classification framework for neuroendocrine neoplasms: an International Agency for Research on Cancer (IARC) and World Health Organization (WHO) expert consensus proposal. Mod Pathol. 2018;31(12):1770-86. https:// doi.org/10.1038/s41379-018-0110-y.

7. Brabander T, van der Zwan WA, Teunissen JJM, Kam BLR, Feelders RA, de Herder WW, et al. Long-term efficacy, survival, and safety of [(177)Lu-DOTA(0),Tyr(3)]octreotate in patients with gastroenteropancreatic and bronchial neuroendocrine tumors. Clin Cancer Res. 2017;23(16):4617-24. https://doi.org/10. 1158/1078-0432.CCR-16-2743. Large safety and efficacy 
analysis of bronchial and GEP NET patients who were treated with ${ }^{177} \mathrm{Lu}$-DOTATATE in a prospective phase II trial.

8. Sabet A, Haug AR, Eiden C, Auernhammer CJ, Simon B, Bartenstein P, et al. Efficacy of peptide receptor radionuclide therapy with (177)Lu-octreotate in metastatic pulmonary neuroendocrine tumors: a dual-centre analysis. Am J Nucl Med Mol Imag. 2017;7(2):74-83.

9. Parghane RV, Talole S, Prabhash K, Basu S. Clinical response profile of metastatic/advanced pulmonary neuroendocrine tumors to peptide receptor radionuclide therapy with $177 \mathrm{Lu}$ DOTATATE. Clin Nucl Med. 2017;42(6):428-35. https://doi. org/10.1097/RLU.0000000000001639.

10. Mariniello A, Bodei L, Tinelli C, Baio SM, Gilardi L, Colandrea $M$, et al. Long-term results of PRRT in advanced bronchopulmonary carcinoid. Eur J Nucl Med Mol Imaging. 2016;43(3):441-52. https://doi.org/10.1007/s00259-015-3190-7.

11. Ianniello A, Sansovini M, Severi S, Nicolini S, Grana CM, Massri $\mathrm{K}$, et al. Peptide receptor radionuclide therapy with (177)LuDOTATATE in advanced bronchial carcinoids: prognostic role of thyroid transcription factor 1 and (18)F-FDG PET. Eur J Nucl Med Mol Imaging. 2016;43(6):1040-6. https://doi.org/10.1007/ s00259-015-3262-8.

12. Lim LE, Chan DL, Thomas D, Du Y, Tincknell G, Kuchel A, et al. Australian experience of peptide receptor radionuclide therapy in lung neuroendocrine tumours. Oncotarget. 2020;11(27):2636-46. https://doi.org/10.18632/oncotarget.27659. This publication described the largest lung NET patient population that was treated with ${ }^{177}$ Lu-DOTATATE only.

13. Zandee WT, Feelders RA, Smit Duijzentkunst DA, Hofland J, Metselaar RM, Oldenburg RA, et al. Treatment of inoperable or metastatic paragangliomas and pheochromocytomas with peptide receptor radionuclide therapy using 177Lu-DOTATATE. Eur J Endocrinol. 2019;181(1):45-53. https://doi.org/10.1530/EJE-180901.

14. Vyakaranam AR, Crona J, Norlen O, Granberg D, Garske-Roman U, Sandstrom M, et al. Favorable outcome in patients with pheochromocytoma and paraganglioma treated with (177)LuDOTATATE. Cancers (Basel). 2019;11:7. https://doi.org/10. 3390/cancers11070909.

15. Nagtegaal ID, Odze RD, Klimstra D, Paradis V, Rugge M, Schirmacher P, et al. The 2019 WHO classification of tumours of the digestive system. Histopathology. 2020;76(2):182-8. https://doi.org/10.1111/his.13975.

16. Dasari A, Shen C, Halperin D, Zhao B, Zhou S, Xu Y, et al. Trends in the incidence, prevalence, and survival outcomes in patients with neuroendocrine tumors in the United States. JAMA Oncol. 2017;3(10):1335-42. https://doi.org/10.1001/jamaoncol. 2017.0589.

17. Heetfeld M, Chougnet CN, Olsen IH, Rinke A, Borbath I, Crespo $\mathrm{G}$, et al. Characteristics and treatment of patients with G3 gastroenteropancreatic neuroendocrine neoplasms. Endocr Relat Cancer. 2015;22(4):657-64. https://doi.org/10.1530/ERC-15-0119.

18. Raj N, Valentino E, Capanu M, Tang LH, Basturk O, Untch BR, et al. Treatment response and outcomes of grade 3 pancreatic neuroendocrine neoplasms based on morphology: well differentiated versus poorly differentiated. Pancreas. 2017;46(3):296-301. https://doi.org/10.1097/MPA.0000000000000735.

19. Sorbye H, Welin S, Langer SW, Vestermark LW, Holt N, Osterlund $\mathrm{P}$, et al. Predictive and prognostic factors for treatment and survival in 305 patients with advanced gastrointestinal neuroendocrine carcinoma (WHO G3): the NORDIC NEC study. Ann Oncol. 2013;24(1):152-60. https://doi.org/10.1093/annonc/mds276.

20. Velayoudom-Cephise FL, Duvillard P, Foucan L, Hadoux J, Chougnet CN, Leboulleux S, et al. Are G3 ENETS neuroendocrine neoplasms heterogeneous? Endocr Relat Cancer. 2013;20(5):649-57. https://doi.org/10.1530/ERC-13-0027.
21. Demirci E, Kabasakal L, Toklu T, Ocak M, Sahin OE, AlanSelcuk N, et al. 177Lu-DOTATATE therapy in patients with neuroendocrine tumours including high-grade (WHO G3) neuroendocrine tumours: response to treatment and long-term survival update. Nucl Med Commun. 2018;39(8):789-96. https://doi.org/ 10.1097/MNM.0000000000000874.

22. Nicolini S, Severi S, Ianniello A, Sansovini M, Ambrosetti A, Bongiovanni $\mathrm{A}$, et al. Investigation of receptor radionuclide therapy with (177)Lu-DOTATATE in patients with GEP-NEN and a high Ki-67 proliferation index. Eur J Nucl Med Mol Imaging. 2018;45(6):923-30. https://doi.org/10.1007/s00259-017-3925-8.

23. Thang SP, Lung MS, Kong G, Hofman MS, Callahan J, Michael $\mathrm{M}$, et al. Peptide receptor radionuclide therapy (PRRT) in European Neuroendocrine Tumour Society (ENETS) grade 3 (G3) neuroendocrine neoplasia (NEN) - a single-institution retrospective analysis. Eur J Nucl Med Mol Imaging. 2018;45(2):26277. https://doi.org/10.1007/s00259-017-3821-2.

24. Zhang J, Kulkarni HR, Singh A, Niepsch K, Muller D, Baum RP. Peptide receptor radionuclide therapy in grade 3 neuroendocrine neoplasms: safety and survival analysis in 69 patients. J Nucl Med. 2019;60(3):377-85. https://doi.org/10.2967/jnumed.118.215848.

25.• Carlsen EA, Fazio N, Granberg D, Grozinsky-Glasberg S, Ahmadzadehfar H, Grana CM, et al. Peptide receptor radionuclide therapy in gastroenteropancreatic NEN G3: a multicenter cohort study. Endocr Relat Cancer. 2019;26(2):227-39. https:// doi.org/10.1530/ERC-18-0424. Multicentre retrospective analysis of 149 GEP NEN grade 3 patients who were treated with PRRT.

26. Pavel M, O'Toole D, Costa F, Capdevila J, Gross D, Kianmanesh $\mathrm{R}$, et al. ENETS consensus guidelines update for the management of distant metastatic disease of intestinal, pancreatic, bronchial neuroendocrine neoplasms (NEN) and NEN of unknown primary site. Neuroendocrinology. 2016;103(2):172-85. https://doi.org/ $10.1159 / 000443167$.

27. Satapathy S, Mittal BR, Sood A, Sood A, Kapoor R, Gupta R. Peptide receptor radionuclide therapy as first-line systemic treatment in advanced inoperable/metastatic neuroendocrine tumors. Clin Nucl Med. 2020;45(9):e393-e9. https://doi.org/10.1097/ RLU.0000000000003170.

28. Rinke A, Müller HH, Schade-Brittinger C, Klose KJ, Barth P, Wied M, et al. Placebo-controlled, double-blind, prospective, randomized study on the effect of octreotide LAR in the control of tumor growth in patients with metastatic neuroendocrine midgut tumors: a report from the PROMID Study Group. J Clin Oncol. 2009;27(28):4656-63. https://doi.org/10.1200/JCO.2009.22. 8510.

29. Caplin ME, Pavel M, Ćwikła JB, Phan AT, Raderer M, Sedláčková E, et al. Lanreotide in metastatic enteropancreatic neuroendocrine tumors. N Engl J Med. 2014;371(3):224-33. https://doi.org/10.1056/NEJMoa1316158.

30. Sowa-Staszczak A, Pach D, Chrzan R, Trofimiuk M, Stefanska A, Tomaszuk M, et al. Peptide receptor radionuclide therapy as a potential tool for neoadjuvant therapy in patients with inoperable neuroendocrine tumours (NETs). Eur J Nucl Med Mol Imaging. 2011;38(9):1669-74. https://doi.org/10.1007/s00259-011-18358.

31. Barber TW, Hofman MS, Thomson BN, Hicks RJ. The potential for induction peptide receptor chemoradionuclide therapy to render inoperable pancreatic and duodenal neuroendocrine tumours resectable. Eur J Surg Oncol. 2012;38(1):64-71. https://doi.org/ 10.1016/j.ejso.2011.08.129.

32. van Vliet EI, van Eijck CH, de Krijger RR, Nieveen van Dijkum EJ, Teunissen JJ, Kam BL, et al. Neoadjuvant treatment of nonfunctioning pancreatic neuroendocrine tumors with $[177 \mathrm{Lu}-$ DOTA0,Tyr3]Octreotate. J Nucl Med. 2015;56(11):1647-53. https://doi.org/10.2967/jnumed.115.158899. 
33. Partelli S, Bertani E, Bartolomei M, Perali C, Muffatti F, Grana $\mathrm{CM}$, et al. Peptide receptor radionuclide therapy as neoadjuvant therapy for resectable or potentially resectable pancreatic neuroendocrine neoplasms. Surgery. 2018;163(4):761-7. https://doi. org/10.1016/j.surg.2017.11.007.

34. Vaughan E, Machta J, Walker M, Toumpanakis C, Caplin M, Navalkissoor S. Retreatment with peptide receptor radionuclide therapy in patients with progressing neuroendocrine tumours: efficacy and prognostic factors for response. Br J Radiol. 2018;91(1091):20180041. https://doi.org/10.1259/bjr.20180041.

35. Yordanova A, Mayer K, Brossart P, Gonzalez-Carmona MA, Strassburg CP, Essler M, et al. Safety of multiple repeated cycles of (177)Lu-octreotate in patients with recurrent neuroendocrine tumour. Eur J Nucl Med Mol Imaging. 2017;44(7):1207-14. https://doi.org/10.1007/s00259-017-3652-1.

36. Pach D, Sowa-Staszczak A, Kunikowska J, Krolicki L, Trofimiuk M, Stefanska A, et al. Repeated cycles of peptide receptor radionuclide therapy (PRRT)-results and side-effects of the radioisotope $90 \mathrm{Y}$-DOTA TATE, $177 \mathrm{Lu}$-DOTA TATE or $90 \mathrm{Y} / 177 \mathrm{Lu}-$ DOTA TATE therapy in patients with disseminated NET. Radiother Oncol. 2012;102(1):45-50. https://doi.org/10.1016/j. radonc.2011.08.006.

37. Rudisile S, Gosewisch A, Wenter V, Unterrainer M, Boning G, Gildehaus FJ, et al. Salvage PRRT with (177)Lu-DOTAoctreotate in extensively pretreated patients with metastatic neuroendocrine tumor (NET): dosimetry, toxicity, efficacy, and survival. BMC Cancer. 2019;19(1):788. https://doi.org/10.1186/s12885019-6000-y.

38. Severi S, Sansovini M, Ianniello A, Bodei L, Nicolini S, Ibrahim T, et al. Feasibility and utility of re-treatment with (177)LuDOTATATE in GEP-NENs relapsed after treatment with (90)YDOTATOC. Eur J Nucl Med Mol Imaging. 2015;42(13):195563. https://doi.org/10.1007/s00259-015-3105-7.

39. Forrer F, Uusijarvi H, Storch D, Maecke HR, Mueller-Brand J. Treatment with $177 \mathrm{Lu}-\mathrm{DOTATOC}$ of patients with relapse of neuroendocrine tumors after treatment with 90Y-DOTATOC. J Nucl Med. 2005;46(8):1310-6.

40. Sabet A, Haslerud T, Pape UF, Sabet A, Ahmadzadehfar H, Grunwald F, et al. Outcome and toxicity of salvage therapy with $177 \mathrm{Lu}$-octreotate in patients with metastatic gastroenteropancreatic neuroendocrine tumours. Eur J Nucl Med Mol Imaging. 2014;41(2):205-10. https://doi.org/10.1007/s00259-013-2547-z.

41.• van der Zwan WA, Brabander T, Kam BLR, Teunissen JJM, Feelders RA, Hofland J, et al. Salvage peptide receptor radionuclide therapy with [(177)Lu-DOTA,Tyr(3)]octreotate in patients with bronchial and gastroenteropancreatic neuroendocrine tumours. Eur J Nucl Med Mol Imaging. 2019;46(3):704-17. https://doi.org/10.1007/s00259-018-4158-1. Safety $(\mathbf{n}=181)$ and efficacy $(n=168)$ analysis of bronchial and GEP NET patients who underwent retreatment and re-retreatment with ${ }^{177} \mathrm{Lu}$ DOTATATE.

42. Ilan E, Sandstrom M, Wassberg C, Sundin A, Garske-Roman U, Eriksson B, et al. Dose response of pancreatic neuroendocrine tumors treated with peptide receptor radionuclide therapy using 177Lu-DOTATATE. J Nucl Med. 2015;56(2):177-82. https:// doi.org/10.2967/jnumed.114.148437.

43. Jahn U, Ilan E, Sandstrom M, Garske-Roman U, Lubberink M, Sundin A. $177 \mathrm{Lu}$-DOTATATE Peptide receptor radionuclide therapy: dose response in small intestinal neuroendocrine tumors. Neuroendocrinology. 2020;110(7-8):662-70. https://doi.org/10. $1159 / 000504001$

44. Sansovini M, Severi S, Ambrosetti A, Monti M, Nanni O, Sarnelli A, et al. Treatment with the radiolabelled somatostatin analog LuDOTATATE for advanced pancreatic neuroendocrine tumors. Neuroendocrinology. 2013;97(4):347-54. https://doi.org/10. $1159 / 000348394$.
45. Garske-Roman U, Sandstrom M, Fross Baron K, Lundin L, Hellman P, Welin S, et al. Prospective observational study of (177)Lu-DOTA-octreotate therapy in 200 patients with advanced metastasized neuroendocrine tumours (NETs): feasibility and impact of a dosimetry-guided study protocol on outcome and toxicity. Eur J Nucl Med Mol Imaging. 2018;45(6):970-88. https://doi. org/10.1007/s00259-018-3945-z. In this large prospective trial, the cumulative PRRT dose was based on kidney and bone marrow dosimetry, allowing half of the NET patient population to receive more than the standard 4 cycles of PRRT.

46. Del Prete M, Buteau FA, Arsenault F, Saighi N, Bouchard LO, Beaulieu A, et al. Personalized (177)Lu-octreotate peptide receptor radionuclide therapy of neuroendocrine tumours: initial results from the P-PRRT trial. Eur J Nucl Med Mol Imaging. 2019;46(3): 728-42. https://doi.org/10.1007/s00259-018-4209-7.

47. Bodei L, Mueller-Brand J, Baum RP, Pavel ME, Horsch D, O'Dorisio MS, et al. The joint IAEA, EANM, and SNMMI practical guidance on peptide receptor radionuclide therapy (PRRNT) in neuroendocrine tumours. Eur J Nucl Med Mol Imaging. 2013;40(5):800-16. https://doi.org/10.1007/s00259-012-2330-6.

48. Riihimaki M, Hemminki A, Sundquist K, Sundquist J, Hemminki $\mathrm{K}$. The epidemiology of metastases in neuroendocrine tumors. Int J Cancer. 2016;139(12):2679-86. https://doi.org/10.1002/ijc. 30400.

49. Braat A, Bruijnen RCG, van Rooij R, Braat M, Wessels FJ, van Leeuwaarde RS, et al. Additional holmium-166 radioembolisation after lutetium-177-dotatate in patients with neuroendocrine tumour liver metastases (HEPAR PLuS): a single-centre, singlearm, open-label, phase 2 study. Lancet Oncol. 2020;21(4):56170. https://doi.org/10.1016/S1470-2045(20)30027-9. In this prospective phase II study, ${ }^{177}$ Lu-DOTATATE was followed by additional radioembolization with Holmium-166 microspheres in 31 grade 1 or 2 NET patients with at least 3 unresectable liver metastases.

50. Ebbers SC, Barentsz MW, Braat AJAT, Lam MGEH. Intraarterial peptide receptor radionuclide therapy for neuroendocrine tumor liver metastases. Digest Dis Interv. 2019;03(01):081-90. https://doi.org/10.1055/s-0038-1676300.

51. Lawhn-Heath C, Fidelman N, Chee B, Jivan S, Armstrong E, Zhang L, et al. Intra-arterial peptide receptor radionuclide therapy using (90)Y-DOTATOC for hepatic metastases of neuroendocrine tumors. J Nucl Med. 2020;62:221-7. https://doi.org/10.2967/ jnumed.119.241273.

52. Ebbers SC, Braat A, Moelker A, Stokkel MPM, Lam M, Barentsz MW. Intra-arterial versus standard intravenous administration of lutetium-177-DOTA-octreotate in patients with NET liver metastases: study protocol for a multicenter, randomized controlled trial (LUTIA trial). Trials. 2020;21(1):141. https://doi.org/10.1186/ s13063-019-3888-0.

53. Cherk MH, Kong G, Hicks RJ, Hofman MS. Changes in biodistribution on (68)Ga-DOTA-Octreotate PET/CT after long acting somatostatin analogue therapy in neuroendocrine tumour patients may result in pseudoprogression. Cancer Imaging. 2018;18(1):3. https://doi.org/10.1186/s40644-018-0136-x.

54. Ayati N, Lee ST, Zakavi R, Pathmaraj K, Al-Qatawna L, Poon A, et al. Long-acting somatostatin analog therapy differentially alters (68)Ga-DOTATATE uptake in normal tissues compared with primary tumors and metastatic lesions. J Nucl Med. 2018;59(2):2237. https://doi.org/10.2967/jnumed.117.192203.

55. Galne A, Almquist $\mathrm{H}$, Almquist M, Hindorf $\mathrm{C}$, Ohlsson $\mathrm{T}$, Nordenstrom E, et al. A prospective observational study to evaluate the effects of long-acting somatostatin analogs on (68)GaDOTATATE uptake in patients with neuroendocrine tumors. J Nucl Med. 2019;60(12):1717-23. https://doi.org/10.2967/ jnumed.119.226332. 
56. Aalbersberg EA, de Wit-van der Veen BJ, Versleijen MWJ, Saveur LJ, Valk GD, Tesselaar MET, et al. Influence of lanreotide on uptake of (68)Ga-DOTATATE in patients with neuroendocrine tumours: a prospective intra-patient evaluation. Eur J Nucl Med Mol Imaging. 2019;46(3):696-703. https://doi.org/10.1007/ s00259-018-4117-x.

57. Yordanova A, Wicharz MM, Mayer K, Brossart P, GonzalezCarmona MA, Strassburg CP, et al. The role of adding somatostatin analogues to peptide receptor radionuclide therapy as a combination and maintenance therapy. Clin Cancer Res. 2018;24(19): 4672-9. https://doi.org/10.1158/1078-0432.CCR-18-0947.

58. Dalm SU, Nonnekens J, Doeswijk GN, de Blois E, van Gent DC, Konijnenberg MW, et al. Comparison of the therapeutic response to treatment with a 177lu-labeled somatostatin receptor agonist and antagonist in preclinical models. J Nucl Med. 2016;57(2): 260-5. https://doi.org/10.2967/jnumed.115.167007.

59. Ginj M, Zhang H, Waser B, Cescato R, Wild D, Wang X, et al. Radiolabeled somatostatin receptor antagonists are preferable to agonists for in vivo peptide receptor targeting of tumors. Proc Natl Acad Sci U S A. 2006;103(44):16436-41. https://doi.org/10. 1073/pnas.0607761103.

60. Cescato R, Waser B, Fani M, Reubi JC. Evaluation of $177 \mathrm{Lu}-$ DOTA-sst2 antagonist versus 177Lu-DOTA-sst2 agonist binding in human cancers in vitro. J Nucl Med. 2011;52(12):1886-90. https://doi.org/10.2967/jnumed.111.095778.

61. Nicolas GP, Mansi R, McDougall L, Kaufmann J, Bouterfa H, Wild D, et al. Biodistribution, pharmacokinetics, and dosimetry of (177)Lu-, (90)Y-, and (111)In-labeled somatostatin receptor antagonist OPS201 in comparison to the agonist (177)LuDOTATATE: the mass effect. J Nucl Med. 2017;58(9):1435-41. https://doi.org/10.2967/jnumed.117.191684.

62. Wild D, Fani M, Fischer R, Del Pozzo L, Kaul F, Krebs S, et al. Comparison of somatostatin receptor agonist and antagonist for peptide receptor radionuclide therapy: a pilot study. J Nucl Med. 2014;55(8):1248-52. https://doi.org/10.2967/jnumed.114.138834.

63. Reidy-Lagunes D, Pandit-Taskar N, O'Donoghue JA, Krebs S, Staton KD, Lyashchenko SK, et al. Phase I trial of welldifferentiated neuroendocrine tumors (NETs) with radiolabeled somatostatin antagonist (177) $\mathrm{Lu}$-satoreotide tetraxetan. Clin Cancer Res. 2019;25(23):6939-47. https://doi.org/10.1158/10780432.CCR-19-1026.

64. Torrisani J, Hanoun N, Laurell H, Lopez F, Maoret JJ, Souque A, et al. Identification of an upstream promoter of the human somatostatin receptor, hSSTR2, which is controlled by epigenetic modifications. Endocrinology. 2008;149(6):3137-47. https://doi.org/ 10.1210/en.2007-1525.

65. Taelman VF, Radojewski P, Marincek N, Ben-Shlomo A, Grotzky A, Olariu CI, et al. Upregulation of key molecules for targeted imaging and therapy. J Nucl Med. 2016;57(11):1805-10. https://doi.org/10.2967/jnumed.115.165092.

66. Veenstra MJ, van Koetsveld PM, Dogan F, Farrell WE, Feelders RA, Lamberts SWJ, et al. Epidrug-induced upregulation of functional somatostatin type 2 receptors in human pancreatic neuroendocrine tumor cells. Oncotarget. 2018;9(19):14791-802. https:// doi.org/10.18632/oncotarget.9462.

67. Jin XF, Auernhammer CJ, Ilhan H, Lindner S, Nolting S, Maurer J, et al. Combination of 5-fluorouracil with epigenetic modifiers induces radiosensitization, somatostatin receptor 2 expression, and radioligand binding in neuroendocrine tumor cells in vitro. J Nucl Med. 2019;60(9):1240-6. https://doi.org/10.2967/jnumed.118.224048.

68. Wanek J, Gaisberger M, Beyreis M, Mayr C, Helm K, Primavesi F, et al. Pharmacological inhibition of class IIA HDACs by LMK235 in pancreatic neuroendocrine tumor cells. Int J Mol Sci. 2018;19(10):3128. https://doi.org/10.3390/ijms19103128.

69. Guenter RE, Aweda T, Carmona Matos DM, Whitt J, Chang AW, Cheng EY, et al. Pulmonary carcinoid surface receptor modulation using histone deacetylase inhibitors. Cancers (Basel). 2019;11(6): 767. https://doi.org/10.3390/cancers1 1060767.

70. Guenter R, Aweda T, Carmona Matos DM, Jang S, Whitt J, Cheng YQ, et al. Overexpression of somatostatin receptor type 2 in neuroendocrine tumors for improved Ga68-DOTATATE imaging and treatment. Surgery. 2020;167(1):189-96. https://doi. org/10.1016/j.surg.2019.05.092.

71. Arvidsson Y, Johanson V, Pfragner R, Wangberg B, Nilsson O. Cytotoxic effects of valproic acid on neuroendocrine tumour cells. Neuroendocrinology. 2016;103(5):578-91. https://doi.org/10. $1159 / 000441849$.

72. Rousseau E, Lau J, Zhang Z, Uribe CF, Kuo HT, Zhang C, et al. Effects of adding an albumin binder chain on $[(177) \mathrm{Lu}] \mathrm{Lu}-$ DOTATATE. Nucl Med Biol. 2018;66:10-7. https://doi.org/10. 1016/j.nucmedbio.2018.08.001.

73. Bandara N, Jacobson O, Mpoy C, Chen X, Rogers BE. Novel Structural Modification Based On Evans Blue Dye To Improve Pharmacokinetics Of A Somastostatin-Receptor-Based Theranostic Agent. Bioconjug Chem. 2018;29(7):2448-54. https://doi.org/10.1021/acs.bioconjchem.8b00341.

74. Zhang J, Wang H, Jacobson O, Cheng Y, Niu G, Li F, et al. Safety, pharmacokinetics, and dosimetry of a long-acting radiolabeled somatostatin analog (177)Lu-DOTA-EB-TATE in patients with advanced metastatic neuroendocrine tumors. J Nucl Med. 2018;59(11):1699-705. https://doi.org/10.2967/jnumed.118.209841.

75. Liu Q, Cheng Y, Zang J, Sui H, Wang H, Jacobson O, et al. Dose escalation of an Evans blue-modified radiolabeled somatostatin analog (177)Lu-DOTA-EB-TATE in the treatment of metastatic neuroendocrine tumors. Eur J Nucl Med Mol Imaging. 2020;47(4): 947-57. https://doi.org/10.1007/s00259-019-04530-1.

76.• Liu Q, Zang J, Sui H, Ren J, Guo H, Wang H, et al. Peptide receptor radionuclide therapy of late-stage neuroendocrine tumor patients with multiple cycles of (177)Lu-DOTA-EB-TATE. J Nucl Med. 2020. https://doi.org/10.2967/jnumed.120.248658. Open-label, non-controlled, non-randomized study investigating the efficacy and safety of ${ }^{177} \mathrm{Lu}$-DOTA-EB-TATE in 32 NET patients.

77. Haug AR, Auernhammer CJ, Wangler B, Schmidt GP, Uebleis C, Goke B, et al. 68Ga-DOTATATE PET/CT for the early prediction of response to somatostatin receptor-mediated radionuclide therapy in patients with well-differentiated neuroendocrine tumors. J Nucl Med. 2010;51(9):1349-56. https://doi.org/10.2967/jnumed. 110.075002 .

78. Gabriel M, Oberauer A, Dobrozemsky G, Decristoforo C, Putzer D, Kendler D, et al. 68Ga-DOTA-Tyr3-octreotide PET for assessing response to somatostatin-receptor-mediated radionuclide therapy. J Nucl Med. 2009;50(9):1427-34. https://doi.org/ 10.2967/jnumed.108.053421.

79. Sharma R, Wang WM, Yusuf S, Evans J, Ramaswami R, Wernig F, et al. (68)Ga-DOTATATE PET/CT parameters predict response to peptide receptor radionuclide therapy in neuroendocrine tumours. Radiother Oncol. 2019;141:108-15. https://doi.org/10. 1016/j.radonc.2019.09.003.

80. Nonnekens J, van Kranenburg M, Beerens CE, Suker M, Doukas $\mathrm{M}$, van Eijck $\mathrm{CH}$, et al. Potentiation of peptide receptor radionuclide therapy by the PARP inhibitor olaparib. Theranostics. 2016;6(11):1821-32. https://doi.org/10.7150/thno.15311.

81. Cullinane C, Waldeck K, Kirby L, Rogers BE, Eu P, Tothill RW, et al. Enhancing the anti-tumour activity of (177)Lu-DOTAoctreotate radionuclide therapy in somatostatin receptor-2 expressing tumour models by targeting PARP. Sci Rep. 2020;10(1): 10196. https://doi.org/10.1038/s41598-020-67199-9.

82. Purohit NK, Shah RG, Adant S, Hoepfner M, Shah GM, Beauregard JM. Potentiation of (177)Lu-octreotate peptide receptor radionuclide therapy of human neuroendocrine tumor cells by 
PARP inhibitor. Oncotarget. 2018;9(37):24693-706. https://doi. org/10.18632/oncotarget.25266.

83. Costa T, Raghavendra NM, Penido C. Natural heat shock protein 90 inhibitors in cancer and inflammation. Eur J Med Chem. 2020;189:112063. https://doi.org/10.1016/j.ejmech.2020.112063.

84. Hofving T, Sandblom V, Arvidsson Y, Shubbar E, Altiparmak G, Swanpalmer J, et al. 177Lu-octreotate therapy for neuroendocrine tumours is enhanced by $\mathrm{Hsp} 90$ inhibition. Endocr Relat Cancer. 2019;26(4):437-49. https://doi.org/10.1530/ERC-18-0509.

85. Lundsten S, Spiegelberg D, Raval NR, Nestor M. The radiosensitizer onalespib increases complete remission in (177)Lu-DOTATATE-treated mice bearing neuroendocrine tumor xenografts. Eur J Nucl Med Mol Imaging. 2020;47(4):98090. https://doi.org/10.1007/s00259-019-04673-1.

86. Ballal S, Yadav MP, Bal C, Sahoo RK, Tripathi M. Broadening horizons with (225)Ac-DOTATATE targeted alpha therapy for gastroenteropancreatic neuroendocrine tumour patients stable or refractory to (177)Lu-DOTATATE PRRT: first clinical experience on the efficacy and safety. Eur J Nucl Med Mol Imaging. 2020;47(4):934-46. https://doi.org/10.1007/s00259-019-045672. Prospective study involving patients with metastatic GEP NET who were treated with ${ }^{225}$ Ac-DOTATATE.

87. Kratochwil C, Giesel FL, Bruchertseifer F, Mier W, Apostolidis C, Boll R, et al. (213)Bi-DOTATOC receptor-targeted alpha-radionuclide therapy induces remission in neuroendocrine tumours refractory to beta radiation: a first-in-human experience. Eur J Nucl Med Mol Imaging. 2014;41(11):2106-19. https://doi.org/ 10.1007/s00259-014-2857-9.

88. Stallons TAR, Saidi A, Tworowska I, Delpassand ES, Torgue JJ. Preclinical Investigation of (212)Pb-DOTAMTATE for peptide receptor radionuclide therapy in a neuroendocrine tumor model. Mol Cancer Ther. 2019;18(5):1012-21. https://doi.org/10.1158/ 1535-7163.MCT-18-1103.

89. Cullinane C, Jeffery CM, Roselt PD, van Dam EM, Jackson S, Kuan K, et al. Peptide receptor radionuclide therapy with (67)CuCuSarTATE is highly efficacious against a somatostatin positive neuroendocrine tumor model. J Nucl Med. 2020;61:1800-5. https://doi.org/10.2967/jnumed.120.243543.

90. Bergsma H, Konijnenberg MW, van der Zwan WA, Kam BL, Teunissen JJ, Kooij PP, et al. Nephrotoxicity after PRRT with (177)Lu-DOTA-octreotate. Eur J Nucl Med Mol Imaging. 2016;43(10):1802-11. https://doi.org/10.1007/s00259-016-3382-9.

91. Bodei L, Kidd M, Paganelli G, Grana CM, Drozdov I, Cremonesi $\mathrm{M}$, et al. Long-term tolerability of PRRT in 807 patients with neuroendocrine tumours: the value and limitations of clinical factors. Eur J Nucl Med Mol Imaging. 2015;42(1):5-19. https://doi. org/10.1007/s00259-014-2893-5.

92. Ahlstedt J, Tran TA, Strand SE, Gram M, Akerstrom B. Human anti-oxidation protein $\mathrm{A} 1 \mathrm{M}-\mathrm{A}$ potential kidney protection agent in peptide receptor radionuclide therapy. Int J Mol Sci. 2015;16(12):30309-20. https://doi.org/10.3390/ijms161226234.

93. Ahlstedt J, Tran TA, Strand F, Holmqvist B, Strand SE, Gram M, et al. Biodistribution and pharmacokinetics of recombinant alpha1-microglobulin and its potential use in radioprotection of kidneys. Am J Nucl Med Mol Imag. 2015;5(4):333-47.

94. Kristiansson A, Ahlstedt J, Holmqvist B, Brinte A, Tran TA, Forssell-Aronsson E, et al. Protection of kidney function with human antioxidation protein alpha1-microglobulin in a mouse (177)Lu-DOTATATE radiation therapy model. Antioxid Redox Signal. 2019;30(14):1746-59. https://doi.org/10.1089/ars.2018. 7517.

95. Andersson CK, Shubbar E, Schuler E, Akerstrom B, Gram M, Forssell-Aronsson EB. Recombinant alpha1-microglobulin is a potential kidney protector in (177)Lu-octreotate treatment of neuroendocrine tumors. J Nucl Med. 2019;60(11):1600-4. https://doi.org/10.2967/jnumed.118.225243.

96. Bodei L, Kidd M, Modlin IM, Severi S, Drozdov I, Nicolini S, et al. Measurement of circulating transcripts and gene cluster analysis predicts and defines therapeutic efficacy of peptide receptor radionuclide therapy (PRRT) in neuroendocrine tumors. Eur J Nucl Med Mol Imaging. 2016;43(5):839-51. https://doi.org/10. 1007/s00259-015-3250-z.

97. Bodei L, Kidd MS, Singh A, van der Zwan WA, Severi S, Drozdov IA, et al. PRRT genomic signature in blood for prediction of (177)Lu-octreotate efficacy. Eur J Nucl Med Mol Imaging. 2018;45(7):1155-69. https://doi.org/10.1007/s00259-018-3967-6.

98. Kwekkeboom DJ, de Herder WW, Kam BL, van Eijck CH, van Essen M, Kooij PP, et al. Treatment with the radiolabeled somatostatin analog [177 Lu-DOTA 0,Tyr3]octreotate: toxicity, efficacy, and survival. J Clin Oncol. 2008;26(13):2124-30. https://doi.org/ 10.1200/JCO.2007.15.2553.

99. Oksuz MO, Winter L, Pfannenberg C, Reischl G, Mussig K, Bares R, et al. Peptide receptor radionuclide therapy of neuroendocrine tumors with (90)Y-DOTATOC: is treatment response predictable by pretherapeutic uptake of (68)Ga-DOTATOC? Diagn Interv Imag. 2014;95(3):289-300. https://doi.org/10.1016/j.diii.2013.07.006.

100. Ambrosini V, Campana D, Polverari G, Peterle C, Diodato S, Ricci C, et al. Prognostic value of $68 \mathrm{Ga}$-DOTANOC PET/CT SUVmax in patients with neuroendocrine tumors of the pancreas. J Nucl Med. 2015;56(12):1843-8. https://doi.org/10.2967/ jnumed.115.162719.

101. Kratochwil C, Stefanova M, Mavriopoulou E, Holland-Letz T, Dimitrakopoulou-Strauss A, Afshar-Oromieh A, et al. SUV of [68Ga]DOTATOC-PET/CT Predicts response probability of PRRT in neuroendocrine tumors. Mol Imaging Biol. 2015;17(3): 313-8. https://doi.org/10.1007/s11307-014-0795-3.

102. Soydal C, Peker A, Ozkan E, Kucuk ON, Kir MK. The role of baseline Ga-68 DOTATATE positron emission tomography/ computed tomography in the prediction of response to fixeddose peptide receptor radionuclide therapy with $\mathrm{Lu}-177$ DOTATATE. Turk J Med Sci. 2016;46(2):409-13. https://doi. org/10.3906/sag-1412-11.

103. Wetz C, Genseke P, Apostolova I, Furth C, Ghazzawi S, Rogasch JMM, et al. The association of intra-therapeutic heterogeneity of somatostatin receptor expression with morphological treatment response in patients undergoing PRRT with $[177 \mathrm{Lu}]$ DOTATATE. PLoS One. 2019;14(5):e0216781. https://doi.org/ 10.1371/journal.pone.0216781.

104. Werner RA, Lapa C, Ilhan H, Higuchi T, Buck AK, Lehner S, et al. Survival prediction in patients undergoing radionuclide therapy based on intratumoral somatostatin-receptor heterogeneity. Oncotarget. 2017;8(4):7039-49. https://doi.org/10.18632/ oncotarget.12402.

105. Graf J, Pape UF, Jann H, Denecke T, Arsenic R, Brenner W, et al. Prognostic significance of somatostatin receptor heterogeneity in progressive neuroendocrine tumor treated with Lu-177 DOTATOC or Lu-177 DOTATATE. Eur J Nucl Med Mol Imaging. 2020;47(4):881-94. https://doi.org/10.1007/s00259019-04439-9.

106. Werner RA, Ilhan H, Lehner S, Papp L, Zsoter N, Schatka I, et al. Pre-therapy somatostatin receptor-based heterogeneity predicts overall survival in pancreatic neuroendocrine tumor patients undergoing peptide receptor radionuclide therapy. Mol Imaging Biol. 2019;21(3):582-90. https://doi.org/10.1007/s11307-018-1252-5.

Publisher's Note Springer Nature remains neutral with regard to jurisdictional claims in published maps and institutional affiliations. 